Homology, Homotopy and Applications, vol.20(1), 2018, pp.259-274

\title{
INTERSECTING THE DIMENSION AND SLICE FILTRATIONS FOR MOTIVES
}

\author{
MIKHAIL V. BONDARKO \\ (communicated by Charles A. Weibel)
}

\begin{abstract}
In this note we prove that the intersections of the levels of the dimension filtration on Voevodsky's motivic complexes over a field with the levels of the slice filtration are "as small as possible". This statement is applied to prove that a conjecture of Ayoub is equivalent to a certain orthogonality assumption.
\end{abstract}

\section{Introduction}

The slice and the dimension filtrations for (various versions of) Voevodsky motives are quite well-known (and easy to define); yet our understanding of motives of dimensions $>1$ is quite limited. In the current note we find out which Voevodsky (effective) motivic complexes of dimension at most $m$ are divisible by the $i$ th power $R\langle i\rangle=R(i)[2 i]$ of the Lefschetz motif (and so, belong to the $i$ th level of the slice filtration; here $R$ is the coefficient ring). Since $R\langle i\rangle$ is a direct summand of the motif $\mathcal{M}_{R}\left(\mathbb{P}^{i}\right)$ of $\mathbb{P}^{i}$, the corresponding intersection of object classes certainly contains motivic complexes of dimension at most $m-i$ tensored by $R\langle i\rangle$ (this class is zero if $m<i$ ), and we prove that this inclusion is actually an equality. This result (Theorem 2.2) is completely new. We use our result (for motives with rational coefficients) to prove that Conjecture 4.22 of [Ayo15] is equivalent to several other assumptions.

Now we describe our results and methods in more detail. Our main object of study is the (monoidal) triangulated category $D M_{R}^{\text {eff }}$ of (unbounded) $R$-linear motivic complexes over a perfect field $k$, where $R$ is any coefficient ring in which the characteristic of $k$ is invertible if it is positive. The category $d_{\leqslant m} D M_{R}^{\text {eff }}$ is the localizing subcategory of $D M_{R}^{\text {eff }}$ generated by the motives of (smooth) varieties of dimension at most $m$. Since for any $i \geqslant 0$ the motif $R\langle i\rangle$ is a direct summand of $\mathcal{M}_{R}\left(\mathbb{P}^{i}\right)$, the category $d_{\leqslant m} D M_{R}^{\text {eff }}$ contains $d_{\leqslant m-i} D M_{R}^{\text {eff }}\langle i\rangle=d_{\leqslant m-i} D M_{R}^{\text {eff }} \otimes_{D M_{R}^{\text {eff }}} R\langle i\rangle$. Recall here that the functor $-\langle i\rangle=-\otimes_{D M_{R}^{\text {eff }}} R\langle i\rangle: M \mapsto M\langle i\rangle$ is a full embedding of $D M_{R}^{\text {eff }}$ into itself (see $[\mathrm{BeV} 08, \S 6.1]$ ); by convention, $d_{\leqslant j} D M_{R}^{\text {eff }}=\{0\}$ if $j<0$. The question is whether the intersection of $d_{\leqslant m} D M_{R}^{\text {eff }}$ with $D M_{R}^{\text {eff }}\langle i\rangle$ (i.e., with the $i$ th level of the slice filtration for $\left.D M_{R}^{\text {eff }}\right)$ equals the "obvious candidate" $d_{\leqslant m-i} D M_{R}^{\text {eff }}\langle i\rangle$.

Research is supported by the Russian Science Foundation grant No. 16-11-10200.

Received June 16, 2017, revised November 13, 2017; published on February 21, 2018.

2010 Mathematics Subject Classification: Primary: 14C15, 18E30. Secondary: 19E15, 14C25, 18 E35.

Key words and phrases: Voevodsky motive, dimension filtration, slice filtration, weight structure, triangulated category, Chow motive.

Article available at http://dx.doi.org/10.4310/HHA.2018.v20.n1.a16

Copyright (C) 2018, International Press. Permission to copy for private use granted. 
Remark. In Voevodsky's notation, our category $d_{\leqslant m-i} D M_{R}^{\text {eff }}\langle i\rangle$ is written as $d_{\leqslant m-i} D M_{R}^{\mathrm{eff}}(i)$.

The starting point of our arguments is that these categories are endowed with so-called Chow weight structures (that are closely related to Chow weight structures introduced in [Bon10] and [Bon11]). Recall here that weight structures on triangulated categories were introduced in [Bon10] (and independently in [Pau08]; one may say that weight structures are certain "cousins" of $t$-structures), whereas Chow weight structures on various triangulated categories of motives are closely related to Deligne's weights of their cohomology.

Combing this observation with the results of [Bon15] we prove that all Chowbounded below objects (i.e., those whose "weights" are bounded from below; here we use the homological convention for the numeration of weights) of $d_{\leqslant m} D M_{R}^{\text {eff }} \cap$ $D M_{R}^{\mathrm{eff}}\langle i\rangle$ belong to $d_{\leqslant m-i} D M_{R}^{\mathrm{eff}}\langle i\rangle$.

This statement is an application of the abstract Proposition 1.9. Moreover, the argument described in Remark 2.3(2) demonstrates that the ("two-sided") bounded case of this statement can be proved using the properties of the weight complex functor that were proved in [Bon10]. Furthermore, all objects of $d_{\leqslant m} D M_{R}^{\mathrm{eff}} \cap D M_{R}^{\mathrm{eff}}\langle i\rangle$ become right weight-degenerate in the Verdier quotient $D M_{R}^{\mathrm{eff}} / d_{\leqslant m-i} D M_{R}^{\mathrm{eff}}\langle i\rangle$ (i.e., "their weights are infinitely small" in this localization). We use the latter statement to prove that

$$
d_{\leqslant m} D M_{-, R}^{\mathrm{eff}} \cap D M_{-, R}^{\mathrm{eff}}\langle i\rangle=d_{\leqslant m-i} D M_{-, R}^{\mathrm{eff}}\langle i\rangle,
$$

where $D M_{-, R}^{\mathrm{eff}}$ is the $R$-linear version of the category of bounded above motivic complexes (so, this is the category that was originally considered by Voevodsky in [Voe00] and [MVW06, §14], whereas the whole $D M_{R}^{\text {eff }}$ was only introduced in [BeV08, Deg11, CiD15]).

Next we recall that in [Ayo15] Ayoub has introduced several interesting conjectures relating the "slice functors" $\nu \geqslant i$ (and related ones) with the dimension filtration (for $R$ being a $\mathbb{Q}$-algebra). In particular, his Conjecture 4.22 states that the functor $\underline{H o m}_{D M_{R}^{\text {eff }}}(R\langle 1\rangle,-)$ sends $d_{\leqslant m} D M_{R}^{\text {eff }}$ into $d_{\leqslant m-1} D M_{R}^{\text {eff }}$ for any $m \geqslant 0$. Our results easily yield that this statement is fulfilled if and only if the right adjoint $\nu \geqslant 1 \cong$ $\underline{H o m}_{D M_{R}^{\text {eff }}}(R\langle 1\rangle,-)\langle 1\rangle$ to the embedding $D M_{R}^{\text {eff }}\langle 1\rangle \rightarrow D M_{R}^{\text {eff }}$ sends $d_{\leqslant m} D M_{R}^{\text {eff }}$ into itself (see Proposition 2.4 for a more general formulation). We also prove that (both of) these conjectures are equivalent to the non-existence of non-zero morphisms from $D M_{\mathrm{gm}, \mathrm{R}}^{\mathrm{eff}}\langle 1\rangle$ into $d_{\leqslant m} D M_{\mathrm{gm}, \mathrm{R}}^{\mathrm{eff}}$ in the localization $D M_{\mathrm{gm}, \mathrm{R}}^{\mathrm{eff}} / d_{\leqslant m-1} D M_{\mathrm{gm}, \mathrm{R}}^{\mathrm{eff}}$ (so, in this assumption it suffices to consider the category $D M_{\mathrm{gm}, \mathrm{R}}^{\mathrm{eff}} \subset D M_{R}^{\mathrm{eff}}$ of effective geometric motives only).

The original motivation for studying these filtration questions comes from the paper [BoS14], which gave various criteria ensuring that an object $M$ of $D M_{\mathrm{gm}, \mathrm{R}}^{\mathrm{eff}}$ belongs to a given level of a certain filtration on motives (including dimension, slice, weight, and connectivity filtrations). These criteria were formulated in terms of the new Chow-weight homology and cohomology theories, and vastly generalize wellknown results about the decomposition of the diagonal. This motivated the author to study the interaction between these filtrations, and it turned out that weight structures yield convenient general methods for questions of this sort. 


\section{Acknowledgments}

The author is deeply grateful to Prof. J. Ayoub for an interesting discussion of his conjectures, and to the referee for his really helpful remarks.

\section{Weight structures and "compactly purely generated" intersections}

This section is mostly dedicated to recollections; still most of the results of $\S 1.3$ are new.

In $\S 1.1$ we introduce some notation and conventions for (mostly, triangulated) categories; we also recall some basics on compactly generated categories.

In $\S 1.2$ we recall some properties of weight structures.

In $\S 1.3$ we relate weight structures to localizations and prove Proposition 1.9 on "intersections of purely compactly generated subcategories" (this is the basic abstract result of this paper).

\subsection{Notation and basics (on compactly generated categories)}

Assume that $C$ is an additive category and $X, Y \in \operatorname{Obj} C$.

- For a category $C$ and $X, Y \in \operatorname{Obj} C$ we will write $C(X, Y)$ for the set of morphisms from $X$ into $Y$ in $C$.

- For a category $C^{\prime}$ we will write $C^{\prime} \subset C$ if $C^{\prime}$ is a full subcategory of $C$.

- We will say that $X$ is a retract of $Y$ if $\operatorname{id}_{X}$ can be factored through $Y$ (note that if $C$ is triangulated then $X$ is a retract of $Y$ if and only if $X$ is its direct summand).

- Let $\underline{H}$ be an additive subcategory of $C$. Then $\underline{H}$ is said to be retraction-closed in $C$ if it contains all retracts of its objects in $C$. The full subcategory $\operatorname{Kar}_{C}(\underline{H})$ of $C$ (here "Kar" is for Karoubi) whose objects are all $C$-retracts of objects $\underline{H}$ will be called the retraction-closure of $\underline{H}$ in $C$.

- The idempotent completion $\operatorname{Kar}(\underline{H})$ (no lower index) of an additive category $\underline{H}$ is the category of "formal images" of idempotents in $\underline{H}$. So, its objects are the pairs $(A, p)$ for $A \in \operatorname{Obj} \underline{H}, p \in \underline{H}(A, A), p^{2}=p$, and the morphisms are given by the formula

$$
\operatorname{Kar}(\underline{H})\left((X, p),\left(X^{\prime}, p^{\prime}\right)\right)=\left\{f \in B\left(X, X^{\prime}\right): p^{\prime} \circ f=f \circ p=f\right\} .
$$

The correspondence $A \mapsto\left(A, \operatorname{id}_{A}\right)$ (for $A \in \operatorname{Obj} \underline{H}$ ) fully embeds $\underline{H} \operatorname{into} \operatorname{Kar}(\underline{H})$. Besides, $\operatorname{Kar}(\underline{H})$ is idempotent complete, i.e., any idempotent morphism in it yields a direct sum decomposition.

- $\underline{C}$ below will always denote some triangulated category; usually it will be endowed with a weight structure $w$.

- For any $A, B, C \in \operatorname{Obj} \underline{C}$ we will say that $C$ is an extension of $B$ by $A$ if there exists a distinguished triangle $A \rightarrow C \rightarrow B \rightarrow A[1]$.

- A class $B \subset \operatorname{Obj} \underline{C}$ is said to be extension-closed if it is closed with respect to extensions and contains 0 . The smallest extension-closed subclass of Obj $\underline{C}$ containing a given $D \subset \operatorname{Obj} \underline{C}$ is called the extension-closure of $D$. 
- Given a class $D$ of objects of $\underline{C}$ we will write $\langle D\rangle$ for the smallest full retractionclosed triangulated subcategory of $\underline{C}$ containing $D$. We will also call $\langle D\rangle$ the subcategory densely generated by $D$.

- For $X, Y \in \operatorname{Obj} \underline{C}$ we will write $X \perp Y$ if $\underline{C}(X, Y)=\{0\}$. For $D, E \subset \operatorname{Obj} \underline{C}$ we write $D \perp E$ if $X \perp Y$ for all $X \in D, Y \in E$; sometimes we will also say that $D$ is (left) orthogonal to $E$.

- Given $D \subset \operatorname{Obj} \underline{C}$ we will write $D^{\perp}$ for the class

$$
\{Y \in \operatorname{Obj} \underline{C}: X \perp Y \forall X \in D\} .
$$

Dually, ${ }^{\perp} D$ is the class $\{Y \in \operatorname{Obj} \underline{C}: Y \perp X \forall X \in D\}$.

We will need the following simple properties of Verdier localizations.

Lemma 1.1. Let $\underline{E} \subset \underline{D} \subset \underline{C}$ be triangulated categories, $M \in \operatorname{Obj} \underline{C}$. Assume that the Verdier quotient $\underline{C^{\prime}}=\underline{C} / \underline{E}$ exists; denote the localization functor $\underline{C} \rightarrow \underline{C} / \underline{E}$ by $\pi$ (and recall that it is the identity on objects). Then the following statements are valid:

1. The localization $\underline{D}^{\prime}=\underline{D} / \underline{E}$ exists also, and the restriction of $\pi$ to $\underline{D}$ gives a full embedding of $\underline{D} / \underline{E}$ into $\underline{C^{\prime}}$.

2. If Obj $\underline{E} \perp M$ then for any $N \in \operatorname{Obj} \underline{C}$ the map $\underline{C}(N, M) \rightarrow \underline{C^{\prime}}(\pi(N), \pi(M))$ is a bijection.

3. Assume that a right adjoint $F$ to the embedding $\underline{D} \rightarrow \underline{C}$ exists. Then $F$ also yields a (well-defined) functor $\underline{C}^{\prime} \rightarrow \underline{D}^{\prime}$ that is right adjoint to the embedding $\underline{D}^{\prime} \rightarrow \underline{C}^{\prime}$ given by assertion 1. In particular, we have $\pi(F(M))=0$ if and only if $\pi(\operatorname{Obj} \underline{D}) \perp \pi(M)$.

Proof. 1, 2. Obvious from the description of morphisms in Verdier localizations (this description can be found in $\S 2.1$ of [Nee01]).

3. The first part of the assertion is given by Lemma 9.1 .5 of ibid.; to obtain its second part one should just apply the definition of a right adjoint functor.

Now let us assume till the end of this subsection that $\underline{C}$ is a triangulated category closed with respect to (small) coproducts; thus it is idempotent complete according to Proposition 1.6.8 of [Nee01].

We recall a few notions related to this setting.

We will call a subcategory $\underline{D} \subset \underline{C}$ the localizing subcategory generated by some $D \subset \operatorname{Obj} \underline{D}$ (or say that $D$ generates $\underline{D}$ as a localizing subcategory of $\underline{C}$ ) if $\underline{D}$ is the smallest full strict triangulated subcategory of $\underline{C}$ containing $D$ that is closed with respect to coproducts.

Moreover, we will call the smallest strict subclass of $\operatorname{Obj} \underline{C}$ that it closed with respect to (small) coproducts, extensions, the shift [1], and contains $D$ the pre-aisle generated by $D$ (this terminology was essentially introduced in [TLS03]).

An object $M$ of $\underline{C}$ is said to be compact if the functor $\underline{C}(M,-)$ commutes with all small coproducts. We will say that $\underline{C}$ is compactly generated if its full (triangulated) subcategory $\underline{C}^{c}$ of compact objects is essentially small and $\operatorname{Obj} \underline{C}^{c}$ generates $\underline{C}$ as its own localizing subcategory.

For a compactly generated $\underline{C}$ and $\underline{H} \subset \underline{C} \underline{C}^{c}$ will say that $\underline{C}$ is compactly generated by $\underline{H}$ if Obj $\underline{H}$ generates $\underline{C}$ as its own localizing subcategory (also). Recall that the latter 
condition is fulfilled if and only if $\underline{C}^{c}$ is densely generated by Obj $\underline{H}$; see Lemma 4.4.5 of [Nee01].

The following well-known lemma will be applied several times throughout the paper.

Lemma 1.2. Let $D$ be a set of compact objects of $\underline{C}$.

Then for $\underline{D}$ being the localizing subcategory generated by $D$ the following statements are valid:

1. The Verdier quotient category $\underline{C}^{\prime}=\underline{C} / \underline{D}$ exists (i.e., its hom-classes are sets); it is closed with respect to coproducts.

2. The localization functor $\pi: \underline{C} \rightarrow \underline{C^{\prime}}$ respects coproducts and converts compact objects into compact ones.

3. The restriction of $\pi$ to the triangulated subcategory $\underline{C}^{c} \subset \underline{C}$ of compact objects of $\underline{C}$ gives a full embedding of $\underline{C}^{c} /\langle D\rangle$ into $\underline{C}^{\prime}$.

4. If some class $C \subset \mathrm{Obj} \underline{C}$ generates $\underline{C}$ as its own localizing subcategory then $\pi(C)$ generates $\underline{C}^{\prime}$ as its own localizing subcategory.

Proof. Assertions 1-3 easily follow from the results of [Nee01] (cf. Proposition 4.3.1.3 (III.1-2) of [BoS16] for closely related statements).

Indeed, Theorem 8.3.3 of [Nee01] implies that $\underline{D}$ satisfies the Brown representability condition (see Definition 8.2.1 of ibid.). Hence Proposition 9.1.19 of ibid. yields the existence of $\underline{C}^{\prime}$. Moreover, $\pi$ respects coproducts according to Corollary 3.2.11 of ibid. The restriction of $\pi$ to $\underline{C}^{c}$ is a full embedding according to Corollary 4.4.2 of ibid.

To finish the proof of assertion 2 it remains to verify that $\pi(M)$ is compact in $\underline{C}^{\prime}$ for any compact object $M$ of $\underline{C}$. We fix $M$; denote by $\underline{D}^{\prime}$ the localizing subcategory of $\underline{C}$ generated by $D \cup\{M\}$. Then the embedding $\underline{D}^{\prime} \rightarrow \underline{C}$ (also) possesses a right adjoint $F$ according to Theorem 8.4.4 of ibid. Obviously, Obj $\underline{D}^{\perp}$ is closed with respect to $\underline{C}$-coproducts (cf. Proposition 1.2.6(III) of [Bon16a]); hence $F$ respects coproducts according to Proposition 1.3.4(4) of ibid. Next, there is an embedding functor $i: \underline{D^{\prime}} / \underline{D} \rightarrow \underline{C^{\prime}}$ that possesses a right adjoint $F^{\prime}$ according to Lemma 1.1(1,3). $F^{\prime}$ respects coproducts also (here we invoke Corollary 3.2.11 of [Nee01] once again). Thus $i$ respects the compactness of objects (obvious from the adjunction of $i$ with $F^{\prime}$ ). Lastly, the localization $l: \underline{D}^{\prime} \rightarrow \underline{D^{\prime}} / \underline{D}$ respects the compactness of objects according to Theorem 4.4 .9 of ibid.; thus $\pi(M)=i \circ l(M)$ is compact indeed.

Assertions 4 is obvious since $\pi$ respects coproducts (see assertion 2).

\subsection{Weight structures: reminder}

Definition 1.3. I. A pair of subclasses $\underline{C}_{w \leqslant 0}, \underline{C}_{w \geqslant 0} \subset \operatorname{Obj} \underline{C}$ will be said to define a weight structure $w$ for a triangulated category $\underline{C}$ if they satisfy the following conditions:

(i) $\underline{C}_{w \leqslant 0}$ and $\underline{C}_{w \leqslant 0}$ are retraction-closed in $\underline{C}$.

(ii) Semi-invariance with respect to translations. $\underline{C}_{w \leqslant 0} \subset \underline{C}_{w \leqslant 0}[1], \underline{C}_{w \geqslant 0}[1] \subset$ $\underline{C}_{w \geqslant 0}$.

(iii) Orthogonality. $\underline{C}_{w \leqslant 0} \perp \underline{C}_{w \geqslant 0}[1]$. 
(iv) Weight decompositions. For any $M \in \operatorname{Obj} \underline{C}$ there exists a distinguished triangle

$$
X \rightarrow M \rightarrow Y \rightarrow X[1]
$$

such that $X \in \underline{C}_{w \leqslant 0}, Y \in \underline{C}_{w \geqslant 0}[1]$.

We will also need the following definitions.

Definition 1.4. Let $i, j \in \mathbb{Z}$.

1. The full subcategory $\underline{H w} \subset \underline{C}$ whose object class is $\underline{C}_{w=0}=\underline{C}_{w \geqslant 0} \cap \underline{C}_{w \leqslant 0}$ is called the heart of $w$.

2. $\underline{C}_{w \geqslant i}\left(\right.$ resp. $\left.\underline{C}_{w \leqslant i}, \underline{C}_{w=i}\right)$ will denote the class $\underline{C}_{w \geqslant 0}[i]$ (resp. $\left.\underline{C}_{w \leqslant 0}[i], \underline{C}_{w=0}[i]\right)$. We will call $\cup_{i \in \mathbb{Z}} \underline{C}_{w \geqslant i}$ the class of $w$-bounded below objects.

3. The class $\underline{C}_{w \geqslant i} \cap \underline{C}_{w \leqslant j}$ will be denoted by $\underline{C}_{[i, j]}$ (so, it equals $\{0\}$ if $i>j$ ). $\underline{C}^{b} \subset \underline{C}$ will be the category whose object class is the class $\cup_{i, j \in \mathbb{Z}} \underline{C}_{[i, j]}$ of $w$ bounded objects.

4. We will call elements of $\cap_{i \in \mathbb{Z}} \underline{C}_{w \leqslant i}$ right $w$-degenerate ones. $w$ will be called left non-degenerate if $\cap_{l \in \mathbb{Z}} \underline{C}_{w \geqslant l}=\{0\}$.

5. Let $\underline{C}$ and $\underline{C}^{\prime}$ be triangulated categories endowed with weight structures $w$ and $w^{\prime}$, respectively; let $F: \underline{C} \rightarrow \underline{C^{\prime}}$ be an exact functor.

$F$ is said to be right weight-exact (with respect to $\left(w, w^{\prime}\right)$ ) if it maps $\underline{C}_{w \geqslant 0}$ into $\underline{C}_{w^{\prime} \geqslant 0}^{\prime}$. We will say that $F$ is weight-exact if it is also left weight-exact, i.e., if $F\left(\underline{C}_{w \leqslant 0}\right) \subset \underline{C}_{w^{\prime} \leqslant 0}^{\prime}$.

6. Let $\underline{H}$ be a full additive subcategory of a triangulated category $\underline{C}$. We will say that $\underline{H}$ is negative (in $\underline{C}$ ) if $\operatorname{Obj} \underline{H} \perp\left(\cup_{i>0} \operatorname{Obj}(\underline{H}[i])\right)$.

7. We will say that a weight structure $w$ is generated by a class $\mathcal{P} \subset \operatorname{Obj} \underline{C}$ whenever $\underline{C}_{w \geqslant 0}=\left(\cup_{i>0} \mathcal{P}[-i]\right)^{\perp}$.

Remark 1.5. 1. A simple example of a weight structure comes from the stupid filtration on the homotopy category $K(B)$ of cohomological complexes for an arbitrary additive category $B$; see Remark 1.2.3(1) of [BoS18] for more detail.

2. In the current paper we use the "homological convention" for weight structures; it was also used [Bon13, BoS14, Bon15, BoS16, BoS18], whereas in [Bon10] the "cohomological convention" was used. In the latter convention the roles of $\underline{C}_{w \leqslant 0}$ and $\underline{C}_{w \geqslant 0}$ are interchanged, i.e., one considers $\underline{C}^{w \leqslant 0}=\underline{C}_{w \geqslant 0}$ and $\underline{C}^{w \geqslant 0}=\underline{C}_{w \leqslant 0}$.

Besides, in [Bon10] both "halves" of $w$ were required to be additive. Yet this additional restriction is easily seen to follow from the remaining axioms; see Remark 1.2.3(4) of [BoS18].

3. The orthogonality axiom (iii) in Definition 1.3 immediately yields that $\underline{H w}$ is negative in $\underline{C}$. We will formulate a certain converse to this statement below.

4. The "right/left convention" for weight-exactness in part 5 of our definition is coherent with the corresponding convention for $t$-structures (note that right or left weight-exact functors are often right or left $t$-exact with respect to certain $t$-structures; cf. Definition 2.1.2(6) of [Bon16a]). On the other hand, the convention used for weight-degenerate objects in part 4 of the definition is somewhat more "natural". So, 
there is a certain discrepancy between these two conventions: a right weight-exact functor sends left weight-degenerate objects into left weight-degenerate ones.

5. Recall also that Pauksztello has introduced weight structures independently in [Pau08]; he called them co-t-structures.

Let us recall some basic properties of weight structures.

Proposition 1.6. Let $\underline{C}$ be a triangulated category endowed with a weight structure $w$. Then the following statements are valid:

1. $\underline{C}_{w \geqslant 0}=\left(\underline{C}_{w \leqslant-1}\right)^{\perp}$ and $\underline{C}_{w \leqslant-1}={ }^{\perp} \underline{C}_{w \geqslant 0}$.

2. For any $i \leqslant j \in \mathbb{Z}$ the class $\underline{C}_{[i, j]}$ equals the extension-closure of $\cup_{i \leqslant m \leqslant j} \underline{C}_{w=m}$. Moreover, $\underline{C}^{b}$ equals the subcategory of $\underline{C}$ densely generated by $\underline{C}_{w=0}$.

3. Let $M \in \underline{C}_{w \leqslant 0}, N \in \underline{C}_{w \geqslant 0}$, and fix some weight decompositions $X_{1}[1] \rightarrow M[1] \stackrel{f[1]}{\rightarrow}$ $Y_{1}[1]$ and $X_{2} \stackrel{g}{\rightarrow} N \rightarrow Y$ of $M[1]$ and $N$, respectively. Then $Y_{1}, X_{2} \in \underline{C}_{w=0}$ and any morphism from $M$ into $N$ can be presented as $g \circ h \circ f$ for some $h \in$ $\underline{C}\left(Y_{1}, X_{2}\right)$.

4. Assume that $w$ is generated by a class $\mathcal{P} \subset \operatorname{Obj} C$; let $w^{\prime}$ be a weight structure on a triangulated category $\underline{C}^{\prime}$, and let $F: \underline{C} \leftrightarrows \underline{C}^{\prime}: G$ be an adjoint pair of exact functors. Then the following conditions are equivalent:

(i) $F$ is left weight-exact.

(ii) $F(\mathcal{P}) \subset \underline{C}_{w^{\prime} \leqslant 0}^{\prime}$

(iii) $G$ is right weight-exact.

5. There is at most one weight structure $w_{\mathcal{P}}$ generated by a given $\mathcal{P} \subset \operatorname{Obj} \underline{C}$, and $\mathcal{P} \subset \underline{C}_{w_{\mathcal{P}} \leqslant 0}$ if $w_{\mathcal{P}}$ exists.

Proof. The first two assertions were established in [Bon10] (yet pay attention to Remark 1.5(2)!). Assertion 3 is precisely Proposition 1.2.3(9) of [Bon15] (and easily follows from the results of [Bon10] also).

Assertion 4 easily follows from assertion 1; see Remark 2.1.5(3) of [Bon16a] for more detail.

Assertion 5 follows from assertion 1 easily also; cf. [Bon16a, Remark 2.1.5(1)].

We also recall some properties of weight structures that may be called compactly generated ones (recall, however, that weight structures described in Proposition 1.7(1) were called strongly $\aleph_{0}$-generated ones in Remark 4.4.4(1) of [Bon16a], whereas the term "compactly generated" was reserved for a wider class of weight structures; moreover, this weight structure $w$ is said to be class-generated by $\underline{H}$ in [BoS17]).

Proposition 1.7. Assume that $\underline{C}$ is compactly generated by its full negative additive subcategory $\underline{H}$.

1. Then $\underline{C}$ possesses a (unique) weight structure $w$ generated by Obj $\underline{H}$. Moreover, $w$ is left non-degenerate, whereas $\underline{H w}$ equals the retraction-closure of the category of all coproducts of objects of $\underline{H}$ (in $\underline{C})$.

Furthermore, $\underline{C}_{w \geqslant 0}$ equals the pre-aisle $\underline{C}_{+}$generated by $\operatorname{Obj} \underline{H}$.

2. Assume that a couple $\left(\underline{C}^{\prime}, \underline{H}^{\prime}\right)$ satisfies our assumptions on $(\underline{C}, \underline{H})$ also. Then any exact functor $F: \underline{C^{\prime}} \rightarrow \underline{C}$ that respects coproducts and sends $\mathrm{Obj} \underline{H}^{\prime}$ into 
Obj $\underline{H}$ is weight-exact with respect to the weight structure $w^{\prime}$ generated by Obj $\underline{H}^{\prime}$ and $w$.

Proof. 1. $w$ exists according to Theorem 4.5.2(I) of [Bon10] (cf. also the proof of [Bon10, Theorem 4.3.2(III)] where the corresponding description of $\underline{C}_{w \geqslant 0}$ is written down explicitly; weight structures of this type are treated in more detail in [BoS17]). $w$ is easily seen to be left non-degenerate; this fact follows from (the categorical dual to) Corollary 5.4.1(8) of [Bon16a]. $\underline{\mathrm{Hw}}$ is calculated in Theorem 4.5.2(II.1) of [Bon10].

Next, Proposition 1.6(1) implies that $\underline{C}_{w \geqslant 0}$ is closed with respect to extensions and the shift [1]; it is closed with respect to coproducts since objects of $\underline{H}$ are compact. Thus $\underline{C}_{w \geqslant 0}$ contains $\underline{C}_{+}$, and the converse inclusion easily follows from Theorem 4.3.2(V.1) of ibid. combined with Proposition 1.6(2).

2. The left weight-exactness of $F$ follows from Proposition 1.6(4). Its right weightexactness is immediate from (the "furthermore" part of) the previous assertion.

\subsection{On intersections of "purely compactly generated" subcategories}

We call a category $\frac{A}{B}$ the factor of an additive category $A$ by its full additive subcategory $B$ if $\operatorname{Obj}\left(\frac{A}{B}\right)=\operatorname{Obj} A$ and $\left(\frac{A}{B}\right)(X, Y)=A(X, Y) /\left(\sum_{Z \in \operatorname{Obj} B} A(Z, Y) \circ\right.$ $A(X, Z))$.

Proposition 1.8. Let $\underline{D}$ be a triangulated subcategory of $\underline{C}$.

I. Suppose that $w$ induces a weight structure $w_{\underline{D}}$ on $\underline{D}$ (i.e., $\operatorname{Obj} \underline{D} \cap \underline{C}_{w \leqslant 0}$ and $\operatorname{Obj} \underline{D} \cap \underline{C}_{w \geqslant 0}$ give a weight structure for $\left.\underline{D}\right)$. Assume that the Verdier quotient of $\underline{C}$ by $\underline{D}$ exists and denote by $\pi$ the localization functor $\underline{C} \rightarrow \underline{C} / \underline{D}$.

Then the following statements are valid:

1. $w$ induces a weight structure on $\underline{C} / \underline{D}$, i.e., the $\underline{C} / \underline{D}$-retraction-closures of $\pi\left(\underline{C}_{w \leqslant 0}\right)$ and $\pi\left(\underline{C}_{w \geqslant 0}\right)$ give a weight structure $w_{\underline{C} / \underline{D}}$ on $\underline{C} / \underline{D}$ (and so, $\pi$ is weight-exact with respect to $w$ and $\left.w_{\underline{C} / \underline{D}}\right)$.

2. The heart $\underline{H w}_{C / \underline{D}}$ of $w_{\underline{C} / \underline{D}}$ is the retraction-closure of (the natural image of) $\frac{H w}{\underline{H w}_{\underline{D}}}$ in $\underline{C} / \underline{D}$. In particular, for any $M^{0}, N^{0} \in \underline{C}_{w=0}$ the homomorphism $\underline{C}\left(M^{0}, N^{0}\right) \rightarrow \underline{C} / \underline{D}\left(\pi\left(M^{0}\right), \pi\left(N^{0}\right)\right)$ is surjective.

3. For any $M \in \underline{C}_{w \leqslant 0}$ and $N \in \underline{C}_{w \geqslant 0}$ the map $\underline{C}(M, N) \rightarrow \underline{C} / \underline{D}(\pi(M), \pi(N))$ is surjective.

II. Assume that $\underline{C}$ and $\underline{H} \subset \underline{C}$ are as in Proposition 1.7(1), and $\underline{D}$ is the localizing subcategory of $\underline{C}$ generated by an additive subcategory $\underline{H}^{\prime}$ of $\underline{H}$ (cf. Proposition 1.7(2)).

1. Then the weight structure $w$ on $\underline{C}$ given by Proposition $1.7(1)$ restricts to $\underline{D}$.

2. The localization $\underline{C} / \underline{D}$ exists and is closed with respect to coproducts, the localization functor $\pi: \underline{C} \rightarrow \underline{C} / \underline{D}$ respects coproducts, and $\pi(\mathrm{Obj} \underline{H})$ compactly generates $\underline{C} / \underline{D}$.

3. The corresponding category $\pi(\underline{H}) \subset \underline{C} / \underline{D}$ is negative in $\underline{C} / \underline{D}$, and the weight structure $w_{\underline{C} / \underline{D}}$ given by assertion I.1 coincides with the weight structure $w^{\prime}$ on $\underline{C} / \underline{D}$ generated by $\pi(\mathrm{Obj} \underline{H})$ (the existence of the latter weight structure is guaranteed by Proposition 1.7(1) provided that $\pi(\underline{H})$ is negative). 
Proof. I. Assertions 1 and 2 are contained in Proposition 8.1.1 of [Bon10].

Assertion 3 is an easy consequence of the ("in particular" part of the) previous assertion combined with Proposition 1.6(3).

II.1. Proposition 1.7(1) gives a weight structure on $\underline{D}$ and part 2 of that proposition (applied to the embedding $F: \underline{D} \rightarrow \underline{C}$ ) ensures that this weight structure is a restriction of $w$.

2. Immediate from Lemma $1.2(1,2,4)$.

3. According to assertion I.1, $\pi(\operatorname{Obj} \underline{H})$ is contained in the heart of $w_{\underline{C} / \underline{D}}$; hence it is negative (in $\underline{C} / \underline{D}$; see Remark $1.5(3)$ ). Next, $\pi$ is weight-exact with respect to $\left(w, w^{\prime}\right)$ according to Proposition 1.7(2). Applying Proposition 1.6(1) to $w^{\prime}$ we easily obtain that $w^{\prime}=w_{\underline{C} / \underline{D}}$.

Now we combine this statement with a result from [Bon15].

Proposition 1.9. Let $\underline{C}$ and $\underline{H}$ be as in Proposition 1.7(1); let $\underline{H}_{1}, \underline{H}_{2}$, and $\underline{H}_{3}$ be full additive subcategories of $\underline{H}$. Denote by $\underline{C}_{i}$ the localizing subcategories of $\underline{C}$ generated by $\underline{H}_{i}$ (for $i=1,2,3$ ) and assume that any morphism from (an object of) $\underline{H}_{1}$ into $\underline{H}_{2}$ vanishes in the Verdier quotient $\underline{C}^{\prime}$ of $\underline{C}$ by $\underline{C}_{3}$ (note that this quotient exists according to Lemma 1.2(1)).

Then all elements of $\operatorname{Obj} \underline{C}_{1} \cap \operatorname{Obj} \underline{C}_{2}$ become right degenerate in $\underline{C}^{\prime}$ (with respect to the weight structure $w^{\prime}$ given by Proposition 1.8(I.1)).

Moreover, w-bounded below elements of $\operatorname{Obj} \underline{C}_{1} \cap \operatorname{Obj} \underline{C}_{2}$ belong to $\operatorname{Obj} \underline{C}_{3}$.

Proof. Denote the localization functor $\underline{C} \rightarrow \underline{C}^{\prime}$ by $\pi$, and denote the weight structures on $\underline{C}_{1}$ and $\underline{C}_{2}$ generated by $\operatorname{Obj} \underline{H}_{1}$ and $\operatorname{Obj} \underline{H}_{2}$, respectively (see Proposition 1.7(1)) by $w_{1}$ and $w_{2}$. According to Remark 3.1.6(1) of [Bon15], to prove our assertions it suffices to verify that the functor $\pi$ kills all $\underline{C}$-morphisms from $\underline{C}_{1, w_{1}=0}$ into $\underline{C}_{2, w_{2}=0}$. Since $\pi$ respects coproducts (see Lemma 1.2(2)), it remains to recall that $\pi$ kills all $\underline{C}$-morphisms from $\underline{H}_{1}$ into $\underline{H}_{2}$.

Remark 1.10. It certainly suffices to assume that any morphism from an object of $\underline{H}_{1}$ into $\underline{H}_{2}$ factors through some object of $\underline{C}_{3}$ (instead of being killed by $\pi$ ). Actually, these two vanishing conditions are equivalent according to Proposition 1.8(I.2).

\section{Intersecting motivic filtrations and a conjecture of Ayoub}

In this section we intersect the levels of the slice filtration on motives over a perfect field $k$ with that of the dimension filtration.

In $§ 2.1$ we study the intersection of the levels of slice filtration with that of the dimension one.

In $§ 2.2$ we relate our results to Conjecture 4.22 of [Ayo15] to obtain several assumptions equivalent to it (we actually prove a more general result of this sort).

In $\S 2.3$ we prove that our results yield a complete calculation of the intersections in question in the subcategory $D M_{-, R}^{\text {eff }} \subset D M_{R}^{\text {eff }}$ of homotopy $t$-structure bounded above motivic complexes (that was considered in [MVW06]). 


\subsection{Intersecting the dimension and the slice filtrations on unbounded motivic complexes}

We start with some preliminaries and notation for motivic complexes.

In this section $k$ will denote a fixed perfect base field of characteristic $p$, and we set $\mathbb{Z}\left[\frac{1}{p}\right]=\mathbb{Z}$ if $p=0$.

The set of smooth projective varieties over $k$ will be denoted by SmPrVar.

- For $R$ being a fixed unital commutative $\mathbb{Z}\left[\frac{1}{p}\right]$-algebra we consider the $R$-linear motivic categories $D M_{\mathrm{gm}, \mathrm{R}}^{\mathrm{eff}} \subset D M_{R}^{\mathrm{eff}}$. So, $D M_{R}^{\mathrm{eff}}$ is the category of unbounded $R$-motivic complexes over $k$ (see Proposition 1.3.1 of [BoK17]). It is closed with respect to small coproducts (and so, idempotent complete); it is compactly generated by its triangulated subcategory $D M_{\mathrm{gm}, \mathrm{R}}^{\mathrm{eff}}$ of effective geometric motives. Moreover, the $R$-linear motif $\mathcal{M}_{R}(X)$ belongs to $\operatorname{Obj}\left\langle\mathcal{M}_{R}(\mathrm{SmPrVar})\right\rangle$ for any $X \in \mathrm{SmVar}$ (since it suffices to verify this statement in the case $R=\mathbb{Z}\left[\frac{1}{p}\right]$; hence one can apply Corollary 3.5.5 of [Voe00] in the case $p=0$ and Theorem 2.2.1(1) of [Bon11] in the case $p>0$ ); thus $D M_{\mathrm{gm}, \mathrm{R}}^{\mathrm{eff}}$ is densely generated by $\mathcal{M}_{R}(\mathrm{SmPrVar})$. Therefore the set $\mathcal{M}_{R}(\mathrm{SmPrVar})$ compactly generates $D M_{R}^{\text {eff }}$ also.

- $\mathcal{M}_{R}(\mathrm{SmPrVar})$ is a negative subcategory of $D M_{R}^{\text {eff }}$ (according to Corollary 6.7.3 of [BeV08]; cf. also Theorem 5.23 of [Deg08]), and the idempotent completion of $\mathcal{M}_{R}(\mathrm{SmPrVar})$ is the category $\underline{C h o w^{\mathrm{eff}}}$ of effective $R$-linear Chow motives that is also negative in $D M_{R}^{\text {eff }}$. We will use the symbol $w_{C h o w}$ to denote the weight structure on $D M_{R}^{\text {eff }}$ generated by $\underline{C h o w}_{R}^{\text {eff }}$ (see Proposition 1.7(1)).

- We also introduce the following notation: $R\langle 1\rangle$ will denote the $R$-linear Lefschetz object; so, it equals $R(1)[2]$ in the notation of [Voe00]. For $i \geqslant 0$ and $M \in$ $\operatorname{Obj} D M_{R}^{\text {eff }}$ we will write $M\langle i\rangle$ for the object $M \otimes_{D M_{R}^{\text {eff }}}(R\langle 1\rangle)^{\otimes i}$.

Recall that the functor $-\langle i\rangle=-\otimes_{D M_{R}^{\text {eff }}} R\langle i\rangle$ is a full embedding of $D M_{R}^{\text {eff }}$ into itself; thus the essential image $D M_{R}^{\text {eff }}(i)=D M_{R}^{\text {eff }}\langle i\rangle$ of this functor is a full subcategory of $D M_{R}^{\text {eff }}$ that is equivalent to $D M_{R}^{\text {eff }}$.

- Note that $R\langle i\rangle$ is a retract of $\mathcal{M}_{R}\left(\mathbb{P}^{i}\right)$ (for any $i \geqslant 0$ ); thus $\underline{C h o w}_{R}^{\mathrm{eff}}\langle i\rangle \subset \underline{C h o w}_{R}^{\mathrm{eff}}$.

- Now we define two filtrations for $D M_{R}^{\text {eff }}$. The so-called slice (or the effectivity) filtration on $D M_{R}^{\mathrm{eff}}$ is given by $D M_{R}^{\mathrm{eff}}\langle i\rangle$ (recall that $D M_{R}^{\mathrm{eff}}\langle i\rangle=D M_{R}^{\mathrm{eff}}(i)$ ) for $i \geqslant 0$.

- For $m \in \mathbb{Z}$ we will write $d_{\leqslant m} D M_{R}^{\text {eff }}$ for the localizing subcategory of $D M_{R}^{\text {eff }}$ generated by $\left\{\mathcal{M}_{R}(X)\right\}$ for $X$ running through smooth $k$-varieties of dimension at most $m$ (so, this category is zero for $m<0$ ). We note that $d_{\leqslant m} D M_{R}^{\text {eff }}$ is compactly generated by $\left\{\mathcal{M}_{R}(P)\right\}$ for $P$ running through smooth projective $k$-varieties of dimension $\leqslant m$ (see Remark 2.2.3 of [BoS14]).

Remark 2.1. In some papers on the subject (in particular, in [Deg11, §4]) only the case $R=\mathbb{Z}$ is considered; one can easily pass to the case $R=\mathbb{Z}\left[\frac{1}{p}\right]$ or $R$ being a localization of $\mathbb{Z}\left[\frac{1}{p}\right]$ (say, $R=\mathbb{Q}$ ) using the more-or-less standard "localization of coefficients" techniques (cf. Proposition 5.6.2 of [Bon16a]). The reader may certainly restrict himself to these cases (that are quite interesting and non-trivial for themselves). One can also reduce our results for an arbitrary $R$ to that for the 
case $R=\mathbb{Z}\left[\frac{1}{p}\right]$; yet this requires some work on the properties of the "forgetful" functor $D M_{R}^{\text {eff }} \rightarrow D M_{\mathbb{Z}\left[\frac{1}{p}\right]}^{\text {eff }}$. So we prefer to treat the case of a general $R$; the most detailed account on $D M_{R}^{\text {eff }}$ in this setting is (probably) [BeV08, $\S 6$ (cf. also [CiD15, MVW06, BoK17]).

Now we are able to prove the central result of this paper.

Theorem 2.2. For any $i, m \geqslant 0$ any element of $\operatorname{Obj} D M_{R}^{\text {eff }}\langle i\rangle \cap \operatorname{Obj} d_{\leqslant m} D M_{R}^{\text {eff }}$ becomes right weight-degenerate (with respect to the weight structure provided by Proposition 1.8) in the localization $D M_{R}^{\mathrm{eff}} / d_{\leqslant m-i} D M_{R}^{\mathrm{eff}}\langle i\rangle$.

Moreover, any $w_{\text {Chow }}$-bounded below element of $\operatorname{Obj} D M_{R}^{\text {eff }}\langle i\rangle \cap \operatorname{Obj} d_{\leqslant m} D M_{R}^{\text {eff }}$ belongs to $\operatorname{Obj} d_{\leqslant m-i} D M_{R}^{\text {eff }}\langle i\rangle$.

Proof. The proof is an easy application of Proposition 1.9. We take $\underline{C}=D M_{R}^{\mathrm{eff}}$, $\underline{H}=\underline{C h o w}_{R}^{\text {eff }}, \underline{H}_{1} \subset \underline{H}$ being the category of motives of smooth projective varieties of dimension at most $m, \underline{H}_{2}=\underline{C h o w}_{R}^{\text {eff }}\langle i\rangle ; \underline{H}_{3}$ is the category of motives of smooth projective varieties of dimension at most $m-i$ twisted by $\langle i\rangle$ (note that $\underline{H}$ contains $\underline{H}_{i}$ for $\left.i=1,2,3\right)$.

By the virtue of the aforementioned proposition (cf. also Remark 1.10), it suffices to verify that any morphism from $\underline{H}_{1}$ into $\underline{H}_{2}$ factors through $\underline{H}_{3}$. The latter fact is precisely Proposition 2.2.6(2) of [BoS14] (moreover, in the case char $k=0$ the proof of [GoG13, Lemma 3] generalizes to give this statement immediately).

Remark 2.3. 1. Since $R\langle i\rangle$ is a retract of $\mathcal{M}_{R}\left(\mathbb{P}^{i}\right)$, we obviously have $\underline{H}_{3} \subset \underline{H}_{2}$ and $\underline{H}_{3} \subset \underline{H}_{1}$. Hence our theorem describes completely the class of $w_{C h o w}$-bounded below elements of $\operatorname{Obj} D M_{R}^{\text {eff }}\langle i\rangle \cap \operatorname{Obj} d_{\leqslant m} D M_{R}^{\text {eff }}$.

2. Recall that any compact object of $D M_{R}^{\mathrm{eff}}$ is $w_{C h o w}$-bounded; hence one can apply the "moreover" part of our proposition to the calculation of $\operatorname{Obj} D M_{\mathrm{gm}, \mathrm{R}}^{\mathrm{eff}}\langle i\rangle \cap$ $\operatorname{Obj} d_{\leqslant m} D M_{\text {gm,R }}^{\text {eff }}$.

The latter calculation has found important applications in [BoS14]. For this reason we explain how to avoid using (Remark 3.1.6(1) of) [Bon15] in its proof (however, our argument is rather similar to that in loc. cit.). So, we verify under the assumptions of Proposition 1.9 that any $w$-bounded object of $\operatorname{Obj} \underline{C}_{1} \cap \operatorname{Obj} \underline{C}_{2}$ belongs to $\operatorname{Obj} \underline{C}_{3}$.

This argument relies on the theory of weight complexes as introduced in $\S 3$ of [Bon10] (whereas in $\S 2.2$ of [Bon16a] some parts of the theory were exposed more carefully). We recall that to any object $M$ of $\underline{C}^{\prime}$ (for $\underline{C^{\prime}}=\underline{C} / \underline{C}_{3}$ ) there is associated its weight complex $t(M) \in \operatorname{Obj} K\left(\underline{H w}^{\prime}\right) ; t(M)$ is well-defined up to homotopy equivalence. The definition of $t(-)$ easily implies that for $i=1$ or 2 and $M \in \pi\left(\operatorname{Obj} \underline{C}_{i}\right)$ the complex $t(M)$ is homotopy equivalent to a complex whose terms belong to $\pi\left(\underline{C}_{i w_{i}=0}\right)$. Since $\pi\left(\underline{C}_{1 w_{1}=0}\right) \perp \pi\left(\underline{C}_{2 w_{2}=0}\right)$ (see Remark 1.10), for any $N \in \operatorname{Obj} \underline{C}_{1} \cap \operatorname{Obj} \underline{C}_{2}$ the morphism $\mathrm{id}_{t(\pi(N))}$ is zero in $K\left(\underline{H w}^{\prime}\right)$. Applying Theorem 3.3.1(V) of [Bon10] we conclude that $\pi(N)=0$.

3. Note that in our theorem one cannot replace $D M_{R}^{\text {eff }} \subset D M_{R}$ by the corresponding version of the motivic stable homotopy category $S H(k)$ (say, for $R$ equal to $\mathbb{Z}$ or to $\mathbb{Z}\left[\mathcal{S}^{-1}\right]$ for $\mathcal{S}$ being a set of primes; then one can define the $R$-linear version of $S H(k)$ as a certain localization). One of the reasons for this is that there is no Chow weight structure on $S H^{c}(k) \subset S H(k)$ (and on $S H^{c}(k)\left[\mathcal{S}^{-1}\right]$ if $2 \notin \mathcal{S}$; see Remark 3.1.2 of [Bon16b] and Remark 6.3.1(3) of [Bon13]). 
Moreover, even the "compact version" of Theorem 2.2 does not carry over to the $S H(k)$-setting. There probably exist plenty of examples illustrating the latter statement. Here we will only note that for $k$ being any formally real field the corresponding category $d_{\leqslant 1} S H^{\text {eff }}(k)$ contains a non-zero compact infinitely effective object (i.e., an element of $\left.\cap_{i \geqslant 0} \operatorname{Obj} S H^{\text {eff }}(k)\langle i\rangle\right)$. Indeed, for the object $C$ constructed in Remark 2.1.2(3) of [Bon16b] we surely have $C\langle 1\rangle \in \operatorname{Obj}_{\leqslant 1} S H^{\mathrm{eff}}(k)$ and $C \neq 0$ in $S H^{\text {eff }}(k)\left[\mathcal{S}^{-1}\right]$ unless $3 \in \mathcal{S}$ (and 3 may be replaced by any other odd prime here). Yet the associated motif $M_{k}(C)$ of $C$ is zero according to loc. cit.; hence $C$ is infinitely effective in $S H^{\text {eff }}(k)$ by Theorem 3.1.1 of ibid.

\subsection{An application to a conjecture of Ayoub}

We recall some basics on "slice" functors.

For any $i \geqslant 0$ the right adjoint to the functor $-\langle i\rangle: D M_{R}^{\mathrm{eff}} \rightarrow D M_{R}^{\mathrm{eff}}$ can certainly be described as $\underline{H o m}_{D M_{R}^{\text {eff }}}(R\langle i\rangle,-)$; this functor respects small coproducts. Next, the composition $\nu^{\geqslant i}=\langle i\rangle \circ \underline{H o m}_{D M_{R}^{\text {eff }}}(R\langle i\rangle,-): D M_{R}^{\text {eff }} \rightarrow D M_{R}^{\text {eff }}$ equals the composition of the embedding $D M_{R}^{\text {eff }}\langle i\rangle \rightarrow D M_{R}^{\text {eff }}$ with the right adjoint to it (immediately from Proposition 4.11 of [Ayo15]).

Now we establish some new properties of the slice functors.

Proposition 2.4. Fix $i, m \geqslant 0$ (along with $R$ ). Then the following statements are valid:

I. $\nu \geqslant i$ is right $w_{\text {Chow }}$-exact.

II. The following conditions are equivalent:

1. $\nu \geqslant i$ sends $d_{\leqslant m} D M_{R}^{\mathrm{eff}}$ into itself.

2. $\underline{H o m}_{D M_{R}^{\mathrm{eff}}}(R\langle i\rangle,-)$ sends $d_{\leqslant m} D M_{R}^{\mathrm{eff}}$ into $d_{\leqslant m-i} D M_{R}^{\mathrm{eff}}$.

3. $\operatorname{Obj} D M_{R}^{\text {eff }}\langle i\rangle$ becomes orthogonal to $\operatorname{Obj} d_{\leqslant m} D M_{R}^{\text {eff }}$ in $D M_{R}^{\text {eff }} / d_{\leqslant m-i} D M_{R}^{\text {eff }}\langle i\rangle$.

4. For any smooth projective $P, Q / k$ and $n \in \mathbb{Z}$ with $\operatorname{dim} Q \leqslant m$ the image of $\mathcal{M}_{R}(P)\langle i\rangle$ in $D M_{R}^{\text {eff }} / d_{\leqslant m-i} D M_{R}^{\text {eff }}\langle i\rangle$ is orthogonal to (the image of) $\mathcal{M}_{R}(Q)[n]$.

5. For any $P, Q, n$ as above the image of $\mathcal{M}_{R}(P)\langle i\rangle$ in $D M_{g m, R}^{\mathrm{eff}} / d_{\leqslant m-i} D M_{g m, R}^{\mathrm{eff}}\langle i\rangle$ is orthogonal to (the image of) $\mathcal{M}_{R}(Q)[n]$.

Proof. I. Recall that $\underline{C h o w}_{R}^{\text {eff }}\langle i\rangle \subset \underline{C h o w}_{R}^{\text {eff }}$ (see $\S 2.1$ ); hence the twist functor

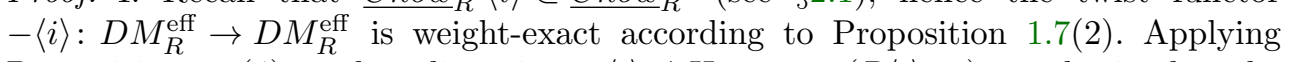
Proposition 1.6(4) to the adjunction $-\langle i\rangle \dashv \underline{\operatorname{Hom}}_{D M_{R}^{\text {eff }}}(R\langle i\rangle,-)$ we obtain that the functor $\underline{H o m}_{D M_{R}^{\text {eff }}}(R\langle i\rangle,-)$ is right $w_{C h o w}$-exact. It remains to note that the composition of right weight-exact functors is right weight-exact also.

II. Condition II.2 implies condition II.1 due to the weight-exactness of $-\langle i\rangle: D M_{R}^{\text {eff }}$ $\rightarrow D M_{R}^{\text {eff }}$; cf. the proof of assertion I.

Next, recall that $d_{\leqslant m} D M_{R}^{\text {eff }}$ is generated by $\left\{\mathcal{M}_{R}(P)\right\}$ for $P$ running through smooth projective $k$-varieties of dimension $\leqslant m$, as a localizing subcategory of $D M_{R}^{\text {eff }}$. Hence to verify the converse implication it suffices to check whether condition II.1 implies that $\underline{H o m}_{D M_{R}^{\text {eff }}}\left(R\langle i\rangle, \mathcal{M}_{R}(P)\right) \in d_{\leqslant m-i} D M_{R}^{\text {eff }}$ if $P$ is smooth projective of dimension at most $m$. Now, $\nu \geqslant i\left(\mathcal{M}_{R}(P)\right) \in D M_{R}^{\text {eff }} w_{C h o w} \geqslant 0$ according to assertion I. It remains to apply (the "moreover" part of) Theorem 2.2. 
So, the first two conditions are equivalent to the assumption that $\nu \geqslant i\left(d_{\leqslant m} D M_{R}^{\mathrm{eff}}\right) \subset$ $d_{\leqslant m-i} D M_{R}^{\text {eff }}\langle i\rangle$. The latter assertion is equivalent to condition II.3 according to Lemma 1.1(3).

Next, Lemma 1.2(2) allows us to verify the orthogonality in condition II.3 only for the images $\pi\left(\mathcal{M}_{R}(P)\langle i\rangle\right)$ and $\pi\left(\mathcal{M}_{R}(Q)[n]\right)$ for $P, Q$, and $n$ as in condition II.4. Hence condition II.3 is equivalent to II.4 according to Lemma 1.2(3). This (part of the) lemma also implies that condition II.4 is equivalent to condition II.5.

Remark 2.5. Note that in the case where $R$ is a $\mathbb{Q}$-algebra and $i=1$ our condition II.2 is exactly Conjecture 4.22 of [Ayo15]. Certainly (for any fixed $R$ ) if condition II.2 is fulfilled for $i=1$ and all $m \geqslant 0$ then it is also fulfilled for all $(i, m)$.

Recall also that certain cases of our condition II.2 were verified in Proposition 4.25 of ibid.

\subsection{Computing intersections inside Voevodsky's $D M_{-, R}^{\text {eff }}$}

Now we extend the "moreover" part Theorem 2.2 to a wider class of objects. We start from a few remarks.

Remark 2.6. 1. The problem with our arguments is that weight structures do not say much on (right) weight-degenerate objects. Note here that non-zero right $w_{C h o w^{-}}$ degenerate objects in $D M_{R}^{\text {eff }}$ do exist (at least) whenever $k$ is a big enough field and $R$ is not a torsion ring (see Remark 2.3.5(3) of [Bon15] that relies on Lemma 2.4 of [Ayo15]).

We also note that this Ayoub's motif belongs to $D M_{R}^{\text {eff }} t_{h o m} \leqslant 0$ (see below), is infinitely effective (i.e., belongs to $\mathrm{Obj} D M_{R}^{\mathrm{eff}}\langle r\rangle$ for all $r \geqslant 0$ ), and its Betti realization vanishes (as proved in loc. cit.).

The author suspects that all $w_{C h o w}$-degenerate objects of $D M_{R}^{\text {eff }}$ are infinitely effective.

2. Starting from the first motivic papers of Voevodsky one of the main tools of working with motivic complexes was the so-called homotopy $t$-structure $t_{\text {hom }}$. Actually, instead of the unbounded category $D M_{R}^{\text {eff }}$ he essentially considered (see $\S 14$ of [MVW06]; the case $R=\mathbb{Z}$ was treated in [Voe00]) its $t_{\text {hom }}$-bounded above subcategory $D M_{-, R}^{\text {eff }}$ whose objects are $\cup_{i \in \mathbb{Z}} D M_{R}^{\text {eff } t_{h o m} \leqslant i}$ (so, we use the cohomological convention for $t$-structures here; cf. [Bon10, §4.1] or [Bon15, §1.4] for more detail on it). Thus the intersection result that we will prove below is completely satisfactory from this older point of view.

Now we recall a description of $t_{\text {hom }}$ that will be convenient for our purposes. According to Theorem 2.4.3 and Example 2.3.5(1) of [BoD17] (where the assumptions on $R$ are the same as in this paper, but the convention for $t$-structures is the homological one), $D M_{R}^{\text {eff } t_{h o m} \leqslant 0}$ is the pre-aisle generated by $\cup_{i \geqslant 0} \operatorname{Obj} \underline{C h o w}_{R}^{\text {eff }}\langle i\rangle[-i]$. Certainly, $D M_{R}^{\text {eff } t_{h o m} \geqslant 0}$ can be recovered from $D M_{R}^{\text {eff } t_{h o m} \leqslant 0}$ using the orthogonality condition (still we will not use this fact below).

We will need the following statement.

Lemma 2.7. For any $m \geqslant 0$ we have $\operatorname{Obj} D M_{R}^{\mathrm{eff}}\langle m+1\rangle \perp \operatorname{Obj} d_{\leqslant m} D M_{R}^{\mathrm{eff}}$.

Proof. In the case where $R$ is a $\mathbb{Q}$-algebra this statement is essentially contained in Proposition 4.25 of [Ayo15]. Moreover, most of the arguments used in the proof of 
loc. cit. can be easily carried over to the case of a general ( $\mathbb{Z}\left[\frac{1}{p}\right]$-algebra) $R$; this yields a reduction of our assertion to the case $m=0$.

Thus it remains to prove that $\operatorname{Obj} D M_{R}^{\text {eff }}\langle 1\rangle \perp \operatorname{Obj}_{\leqslant 0} D M_{R}^{\text {eff }}$. It certainly suffices to check that $\mathcal{M}_{R}(X)\langle 1\rangle \perp \mathcal{M}_{R}(Y)[i]$ for any $i \in \mathbb{Z}$ and smooth $X, Y / k$ with $Y$ of dimension zero. Since $Y$ is also proper, the object $\mathcal{M}_{R}(Y)$ is self-dual in the tensor category $D M_{R}^{\text {eff }}$ (immediately from the duality statement given by Proposition 6.7.1 of [BeV08]). Hence the orthogonality in question reduces to $\mathcal{M}_{R}(X \times Y)\langle 1\rangle \perp \mathcal{M}_{R}(\operatorname{Spec} k)[i]$; thus it follows from the vanishing mentioned in [BeV08, Corollary 6.1].

Now we are able to prove the following version of Theorem 2.2.

Proposition 2.8. For any $i, m \geqslant 0$ the class $\operatorname{Obj} D M_{-, R}^{\text {eff }}\langle i\rangle \cap \operatorname{Obj} d_{\leqslant m} D M_{R}^{\text {eff }}$ equals $\left(\operatorname{Obj} D M_{-, R}^{\text {eff }} \cap \operatorname{Obj} d_{\leqslant m-i} D M_{R}^{\text {eff }}\right)\langle i\rangle$.

Proof. Certainly, if $N\langle i\rangle \in \operatorname{Obj} D M_{-, R}^{\text {eff }}$ for $N \in \operatorname{Obj} D M_{R}^{\text {eff }}$ then $N$ is also $t_{h^{\prime} m^{-}}$ bounded above also: this is a consequence of the $t$-exactness of the endofunctor $\underline{H o m}_{D M_{R}^{\text {eff }}}(R\langle i\rangle[-i],-)$ (cf. the beginning of $\S 2.2$ ) that is given by Example 1.3.1(6) and Corollary 3.3.7(3) of [BoD17]. Thus it suffices to prove that any $M \in$ $D M_{R}^{\text {eff } t_{\text {hom }} \leqslant 0} \cap \operatorname{Obj} D M_{R}^{\text {eff }}\langle i\rangle \cap \operatorname{Obj} d_{\leqslant m} D M_{R}^{\text {eff }}$ belongs to Obj $d_{\leqslant m-i} D M_{R}^{\text {eff }}\langle i\rangle$.

Now we apply Lemma 1.1(2). We take $\underline{C}=D M_{R}^{\mathrm{eff}}, \underline{E}=D M_{R}^{\mathrm{eff}}\langle m+1\rangle, \underline{C^{\prime}}=\underline{C} / \underline{E}$. By Lemma 2.7, Obj $\underline{E} \perp \operatorname{Obj} d_{\leqslant m} D M_{R}^{\text {eff }}$. Hence the localization functor $\pi: \underline{C} \rightarrow \underline{C}^{\prime}$ restricts to a full embedding of $d_{\leqslant m} D M_{R}^{\text {eff }}$ into $\underline{C}^{\prime}$. Since $d_{\leqslant m-i} D M_{R}^{\text {eff }}\langle i\rangle \subset d_{\leqslant m} D M_{R}^{\text {eff }}$, it suffices to verify that $\pi(M)$ belongs to $\pi\left(\mathrm{Obj} d_{\leqslant m-i} D M_{R}^{\text {eff }}\langle i\rangle\right)$.

Now, the description of $D M_{R}^{\text {eff }} t_{\text {hom }} \leqslant 0$ given in Remark 2.6(2) yields that $\pi(M)$ belongs to the pre-aisle generated by $\cup_{0 \leqslant i \leqslant m} \pi\left(\operatorname{Obj} \underline{C h o w}_{R}^{\mathrm{eff}}\langle i\rangle[-i]\right)$. Thus $\pi(M)$ is bounded below with respect to the weight structure for $\underline{C}^{\prime}$ generated by $\pi\left(\operatorname{Obj} \underline{C h o w}{ }_{R}^{\text {eff }}\right)$ (see Proposition 1.8(II)). Hence the image $M^{\prime}$ of $\pi(M)$ in $\underline{C}^{\prime} / \pi\left(d_{\leqslant m-i} \overline{D M_{R}^{\mathrm{eff}}}\langle i\rangle\right)$ (actually, $M^{\prime}$ is just $M$ considered as an object of $\left.\underline{C}^{\prime} / \pi\left(d_{\leqslant m-i} D M_{R}^{\text {eff }}\langle i\rangle\right)\right)$ is also bounded below with respect to the corresponding weight structure provided by Proposition 1.8 (here we apply parts II.(2-3) of the proposition). On the other hand, $M^{\prime}$ is also right $w_{C h o w}$-degenerate since the image of $M$ in $\underline{C} /\left(d_{\leqslant m-i} D M_{R}^{\mathrm{eff}}\langle i\rangle\right)$ is so according to Theorem 2.2 (here we invoke Proposition 1.8(II.3) once again). Thus $M^{\prime} \perp M^{\prime}$ by the orthogonality axiom of weight structures; hence $M^{\prime}=0$. Therefore $\pi(M)$ does belong to $\pi\left(d_{\leqslant m-i} D M_{R}^{\text {eff }}\langle i\rangle\right)$.

Remark 2.9. 1. Formally (the formulation of) our proposition "depends on $D M_{R}^{\text {eff }}$ " since it treats intersections of certain localizing subcategories of $D M_{R}^{\text {eff }}$ with Obj $D M_{-, R}^{\text {eff }}$. However, the natural $D M_{-, R}^{\text {eff }}$-version of our result (stated in terms the corresponding "localizing" classes of objects in $D M_{-, R}^{\text {eff }}$; those are closed only with respect to those coproducts that exist in $D M_{-, R}^{\text {eff }}$ ) is also true. Indeed, for any $j \geqslant 0$ there exists an (exact) right adjoint to the embeddings $d_{\leqslant j} D M_{R}^{\text {eff }} \rightarrow D M_{R}^{\text {eff }}$ (see Lemma 1.2(1)). Since this functor also respects coproducts (see part 2 of the lemma), it suffices to check that it restricts to an endofunctor of $D M_{-, R}^{\text {eff }}$. The latter assertion was mentioned in the beginning of [Voe00, §3.4] (in the case $R=\mathbb{Z}$ that does not differ from the general one in this matter); respectively, it can also be easily established using the methods of the proof of [BoD17, Corollary 3.7]. 
2. Note that the Chow weight structure on $D M_{R}^{\text {eff }}$ can be restricted to $D M_{-, R}^{\text {eff }}$ (since the arguments of [Bon10, $\S 7.1]$ carry over to our more general setting without any problems). Yet the author was not able to apply this fact for the purposes of the current paper.

3. The main subject of [BoK17] is an interesting candidate for the Chow weight structure on $D M_{R}^{\mathrm{eff}}$ that is defined independently from the assumption that $p$ is invertible in $R$. It is proven that this weight structure satisfies several important properties of $w_{\text {Chow }}$ (in particular, it coincides with $w_{\text {Chow }}$ whenever $R$ is a $\mathbb{Z}\left[\frac{1}{p}\right]$ algebra). Yet it is not clear whether this weight structure may be restricted to the subcategories $d_{\leqslant m} D M_{R}^{\text {eff }}$ (unless it coincides with $w_{C h o w}$ ); so the methods of the current paper cannot be used for the study of $\operatorname{Obj} D M_{R}^{\text {eff }}\langle i\rangle \cap \operatorname{Obj} d_{\leqslant m} D M_{R}^{\text {eff }}$ in this greater generality.

\section{References}

[Ayo15] Ayoub J., Motives and algebraic cycles: a selection of conjectures and open questions, preprint, 2015, http://user.math.uzh.ch/ayoub/ PDF-Files/Article-for-Steven.pdf.

[BeV08] Beilinson A., Vologodsky V. A DG guide to Voevodsky motives, Geom. Funct. Anal. 17(6) (2008), 1709-1787.

[Bon10] Bondarko M.V., Weight structures vs. $t$-structures; weight filtrations, spectral sequences, and complexes (for motives and in general), J. Ktheory 6(3) (2010), 387-504, see also http://arxiv.org/abs/0704. 4003.

[Bon11] Bondarko M.V., $\mathbb{Z}\left[\frac{1}{p}\right]$-motivic resolution of singularities, Compos. Math. 147(5) (2011), 1434-1446.

[Bon13] Bondarko M.V., Gersten weight structures for motivic homotopy categories; direct summands of cohomology of function fields and coniveau spectral sequences, preprint, http://arxiv.org/abs/1312.7493.

[Bon15] Bondarko M.V., On morphisms killing weights, weight complexes, and Eilenberg-Maclane (co)homology of spectra, preprint, http://arxiv . org/abs/1509.08453v4.

[Bon16a] Bondarko M.V., On torsion pairs, (well generated) weight structures, adjacent $t$-structures, and related (co)homological functors, preprint, https://arxiv.org/abs/1611.00754.

[Bon16b] Bondarko M.V., On infinite effectivity of motivic spectra and the vanishing of their motives, preprint, http://arxiv.org/abs/1602.04477.

[BoD17] Bondarko M.V., Déglise F., Dimensional homotopy $t$-structures in motivic homotopy theory, Adv. Math. 311 (2017), 91-189.

[BoK17] Bondarko M.V., Kumallagov D.Z., On Chow weight structures without projectivity and resolution of singularities, to appear in Algebra i Analiz, https://arxiv.org/abs/1711.08454.

[BoS14] Bondarko M.V., Sosnilo V.A., Detecting the $c$-effectivity of motives, their weights, and dimension via Chow-weight (co)homology: a "mixed 
motivic decomposition of the diagonal", preprint, http://arxiv.org/ abs/1411.6354.

[BoS16] Bondarko M.V., Sosnilo V.A., Non-commutative localizations of additive categories and weight structures; applications to birational motives, J. Inst. Math. Jussieu, published online: 24 May 2016, 1-37, DOI: 10.1017/S1474748016000207.

[BoS17] Bondarko M.V., Sosnilo V.A., On purely generated $\alpha$-smashing weight structures and weight-exact localizations, preprint, http://arxiv.org/ abs/1712.00850.

[BoS18] Bondarko M.V., Sosnilo V.A., On constructing weight structures and extending them to idempotent extensions, Homology, Homotopy Appl. 20(1), 2018, 37-57.

[CiD15] Cisinski D.-C., Déglise F., Integral mixed motives in equal characteristic, Doc. Math., Extra Volume: Alexander S. Merkurjev's Sixtieth Birthday (2015), 145-194.

[Deg08] Déglise F., Around the Gysin triangle II, Doc. Math. 13 (2008), 613-675.

[Deg11] Déglise F., Modules homotopiques (Homotopy modules), Doc. Math. 16 (2011), 411-455.

[GoG13] Gorchinskiy S., Guletskij V., Non-trivial elements in the Abel-Jacobi kernels of higher-dimensional varieties, Adv. Math. 241 (2013), 162191.

[MVW06] Mazza C., Voevodsky V., Weibel Ch., Lecture Notes on Motivic Cohomology, Clay Mathematics Monographs, vol. 2, 2006.

[Nee01] Neeman A., Triangulated Categories, Annals of Mathematics Studies, vol. 148, Princeton University Press, 2001, viii+449 pp.

[Pau08] Pauksztello D., Compact cochain objects in triangulated categories and co-t-structures, Cent. Eur. J. Math. 6(1) (2008), 25-42.

[TLS03] Tarrio L.A., Lopez A.J., Salorio M.J., Construction of $t$-structures and equivalences of derived categories, Trans. Amer. Math. Soc. 355(6) (2003), 2523-2543.

[Voe00] Voevodsky V. Triangulated categories of motives over a field, in: Voevodsky V., Suslin A., and Friedlander E., Cycles, Transfers and Motivic Homology Theories, Annals of Mathematical studies, vol. 143, Princeton University Press, 2000, pp. 188-238.

Mikhail V. Bondarko m.bondarko@spbu.ru

St. Petersburg State University, Department of Mathematics and Mechanics, Universitetskij pr. 28, 198904, St. Petersburg, Russia 